\title{
Early Directed Oritavancin Therapy in the Emergency Department May Lead to Hospital Avoidance Compared to Standard Treatment for Acute Bacterial Skin and Skin Structure Infections: A Real-World Retrospective Analysis
}

\author{
Brittany Helton ${ }^{1} \cdot$ Ashley MacWhinnie $^{1} \cdot$ Sarah Brooks Minor ${ }^{1} \cdot$ Thomas P. Lodise $^{2} \cdot$ Kelly D. Rafferty $^{1}$. \\ Steven L. Allison ${ }^{1}$
}

Published online: 25 June 2020

(c) The Author(s) 2020

\begin{abstract}
Objectives Acute bacterial skin and soft tissue infections (ABSSSIs) are a leading cause of presentation to the emergency department (ED). This study aimed to determine the potential impact of utilizing oritavancin in the ED or observation unit (OBS) on hospital inpatient admission.

Methods A single-center community teaching hospital developed a pharmacy-led pilot to evaluate the use of oritavancin as a measure to avoid hospital admissions/readmissions in appropriate patients with ABSSSIs. Prior to initiation of the oritavancin pilot, prespecified inclusion and exclusion criteria were determined for proper patient selection. The pilot ran from January 1 to December 31, 2017. The data were compared to corresponding data for an equal number of patients during the pilot period who had similar ABSSSI diagnoses to the oritavancin pilot group but received vancomycin. The primary outcome was length of stay (LOS), defined as the total time in hours from presentation to the ED until discharge home, including time spent in the OBS or inpatient unit.

Results During the study period, 122 patients met the study criteria and 61 patients received oritavancin in the ED or OBS unit. These patients were compared to 61 consecutive patients during the pilot period who received vancomycin. Administration of oritavancin in the ED or OBS was associated with a significantly shorter mean LOS relative to the standard of care group (19.5 vs. $85.98 \mathrm{~h}, p<0.01)$. All-cause 30-day readmissions were the same for both groups (6 vs. $6, p=1)$.

Conclusions These results suggest that use of oritavancin in the ED or OBS setting for ABSSSIs may shorten LOS without negatively affecting readmissions.
\end{abstract}

\section{Introduction}

Oritavancin is a novel lipoglycopeptide antibiotic that is FDA approved for the treatment of acute bacterial skin and skin structure infections (ABSSSIs) caused by susceptible gram-positive organisms such as Staphylococcus aureus,

Digital features To view digital features for this article go to https://doi.org/10.6084/m9.figshare.12221690.

Brittany Helton

Brittany.helton@adventhealth.com

1 AdventHealth East Orlando Department of Pharmacy, Orlando, FL, USA

2 Albany College of Pharmacy and Health Sciences, Albany, NY, USA
Streptococcus spp., and Enterococcus faecalis [1]. Similar to vancomycin, oritavancin disrupts cell wall synthesis by inhibiting transpeptidation, but it has the additional benefit of increasing membrane permeability via improved binding site affinity [2]. Relative to other antibiotics approved for the treatment of patients with ABSSSIs, the greatest distinguishing feature of oritavancin is its single fixed-dose treatment, with no requirement for therapeutic drug monitoring. In two phase III trials, a single IV dose of $1200 \mathrm{mg}$ of oritavancin had comparable efficacy and safety to 7-10 days of vancomycin for the treatment of adult patients with ABSSSIs [3, 4].

Data indicate that many hospitalized ABSSSI patients have zero or few uncontrolled comorbid conditions and limited to no systemic signs and symptoms of infection, and are often solely admitted to receive IV antibiotics [5, 6]. 


\section{Key Points}

A pharmacy-led criteria-for-use oritavancin pathway was initiated at a community hospital in the emergency department (ED) or observation unit (OBS) as a measure to avert hospital inpatient admissions of certain designated patients with ABSSSIs.

Relative to standard practices, use of oritavancin in the ED or OBS setting for skin and skin structure infections may shorten LOS, defined as the total time in hours from presentation to the ED until discharge home, including time spent in the OBS or inpatient unit.

Early directed oritavancin use was not found to result in increased 30-day subsequent readmissions and 30-day return ED visits relative to standard inpatient treatment with vancomycin.

The mortality rate is low among ABSSSI patients with no comorbidities, and effective outpatient treatment should be encouraged in order to reduce unnecessary costs and the healthcare burden on hospitals [7]. Because of its singledose therapy, it has been postulated that oritavancin could be used to shift the care of appropriate ABSSSI patients from the inpatient to the outpatient setting [8]. Across the SOLO trials, 792 patients were treated solely in the outpatient setting, and very few patients in the oritavancin group $(1.3 \%)$ required subsequent care in the inpatient setting post outpatient treatment [9]. More recently, data from the Clinical and Historic Registry and Orbactiv Medical Evaluation (CHROME) postmarketing registry reported that over $85 \%$ of patients were treated in an outpatient infusion center, a positive clinical response was observed in $92.8 \%$ of patients, and microbial eradication was observed in $90.0 \%$ of patients with posttherapy cultures. Within 28 days following oritavancin administration, only 4 (3.6\%) patients were hospitalized due to failed treatment for the index infection [10].

At AdventHealth East Orlando (AHEO), a 295-bed community teaching hospital, a quality assurance study found that the majority of patients hospitalized at this institution with cellulitis-related diagnoses had an average length of stay (LOS) of 4.13 days and an average cost of care totaling \$5,600 USD per case. Consistent with other studies [5], most hospitalized patients had limited disease severity and comorbid conditions, indicating that many of these patients could have been treated in the outpatient setting. Based on these findings, AHEO developed a pharmacy-led pilot for the use of oritavancin in the emergency department (ED) or observation unit (OBS) as a measure to avert hospital inpatient admissions in appropriate patients with ABSSSIs. The objective of this analysis was to evaluate the effectiveness of the pharmacy-led oritavancin pilot relative to patients with ABSSSI who met the criteria for the oritavancin but received vancomycin during the same timeframe.

\section{Methods}

\subsection{Study Design}

A retrospective cohort analysis of patients who presented to the ED or OBS with a skin and soft tissue infection at AHEO was performed from January 1, 2017 through December 31, 2017. Patients who received oritavancin in the ED or OBS as part of the pharmacy-led pilot (pilot group) were compared to patients who met the criteria for oritavancin use during the study period but received vancomycin therapy (standard of care group).

\subsection{Description of the Pilot Group}

Pharmacists, in collaboration with ED providers, prospectively identified patients who met the prespecified criteria (Appendix A) to receive oritavancin by reviewing the ED track board and assessing the electronic medical record. Once it was confirmed that the patient met the qualifications to receive oritavancin, the pharmacist met with the patient to explain the medication and expectations for treatment. Following patient consent, the pharmacy prepared and dispensed oritavancin to the ED for the 3-h infusion time. Patients were discharged and the AHEO outpatient transitions of the care clinic called each patient within 5 days of discharge. At least three phone calls were placed to attempt to reach the patient, and the pharmacist was trained to instruct the patient to follow up with their physician or return to the ED if symptoms persisted after discharge or were becoming worse. Some patients who underwent incision and drainage were asked to return to the ED within $48 \mathrm{~h}$ for wound care at the physician's discretion.

\subsection{Description of the Standard of Care Group}

The standard of care group comprised patients who met the criteria for oritavancin use during the study period but received vancomycin in the ED and continued with vancomycin therapy. Only patients with an ICD-10 code on the Medicines Company list of indications approved for their drug replacement program were included in the pilot group (Table 5 in Appendix B), so this criterion was used for inclusion in the standard of care group. A list of consecutive patients with these ICD-10 codes from January 1, 2017 through December 31, 2017 were screened. Patients were included in the standard of care group if they received vancomycin and met all study criteria (see the next section). 
The screening of consecutive patients for inclusion in the standard of care group was stopped once there were equal numbers of patients in the standard of care group and the pilot group.

\subsection{Study Criteria}

For both the oritavancin pilot and standard of care groups, the criteria for entry into this analysis was as follows: $\geq 18$ years old; had a proven or suspected gram-positive ABSSSI [11] (i.e., skin infection with a total area of lesion plus surrounding erythema, edema, and/or induration of approximately $\geq 75 \mathrm{~cm}^{2}$ ); and met prespecified criteria for oritavancin use in the ED (Appendix A). Patients were excluded if they were pregnant; presented with sepsis, bacteremia, osteomyelitis, necrotizing fasciitis, endocarditis, or unstable vital signs; had a chronic infection such as diabetic foot infection or ulceration, infected burn, or decubitus ulcer; had a history of hypersensitivity to oritavancin; had a blood glucose level of $\geq 300 \mathrm{mg} / \mathrm{dL}$; or required unfractionated heparin sodium.

\subsection{Data Elements}

Trained reviewers collected data from the patients' electronic medical records. Data elements that were extracted included demographics, comorbidities, length of hospitalization, antimicrobial medications, and causative pathogen for the ABSSSI. Demographic characteristics included age, sex, actual body weight, body mass index (BMI), race, comorbidities, and whether the patient was febrile on admission or met SIRS criteria [12]. The investigators chose to use SIRS criteria in keeping with the institution's sepsis alert criteria, and to correlate with outcomes of the SOLO trials and a previous internal analysis of inpatient use of oritavancin. Comorbidities that were collected included the Charlson Comorbidity Index (CCI) [13] and an active or recent history of IV drug abuse, diabetes, hepatitis, an immunocompromised state (including the presence of human immunodeficiency virus, HIV), renal insufficiency (defined as a glomerular filtration rate of $<50 \mathrm{ml} / \mathrm{min} / 1.73 \mathrm{~m}^{2}$ ) [14], and severe peripheral vascular conditions. These covariates were selected based on those studied in the SOLO trials. For patients with microbiologic cultures, results were assessed for the presence of $S$. aureus and whether the pathogen was methicillin susceptible. Admission to the OBS or inpatient unit and discharge from the ED, OBS, or inpatient unit were all determined by the treating physician; neither the pharmacy nor investigators were involved in this process.

\subsection{Clinical Outcomes}

The following clinical outcomes were assessed: mean index hospital LOS, 30-day subsequent readmissions (all cause and infection related), and 30-day return ED visits (all cause and infection related). LOS was defined as the total time in hours from presentation to the ED until discharge home, including time spent in the OBS or inpatient unit. Readmission included all patients who were admitted to the hospital within 30 days of their initial presentation and treatment and were then assessed for cause of readmission. ED return looked at all patients who returned to the ED within 30 days, regardless of whether they were readmitted to the hospital or discharged home from the ED. For both 30-day subsequent readmissions and return ED visits, patients who returned for any infection were considered to be ID related, whereas those who returned for the index infection were considered to be patients with a recurrent ABSSSI. Patients who returned due to the physician's instructions on wound care were also recorded. Additionally, a follow-up via phone call was conducted for patients in the pilot program to assess their current status, as described previously.

Cost savings were assessed primarily as the number of days of inpatient admission avoided. This number was calculated as the difference in total LOS between all patients in the standard of care group and all patients in the pilot group. The overall cost saved by inpatient admission avoidance was based on an average cost per day of $\$ 1,355.93$ USD as determined by an internal review of the cost per case and the average LOS for the ABSSSI diagnosis-related group (DRG). Insurance reimbursement was recorded during the chart review, as was the number of patients who underwent drug replacement. Cost savings for drug replacement included all patients with a successful drug replacement by the manufacturer, and were determined based on the cost of the medication at this facility.

\subsection{Statistical Analysis Plan}

Categorical variables were compared using Fisher's exact test, and continuous variables were compared using Student's $t$ test or the Mann-Whitney $U$ test. A Kaplan-Meier analysis was used to assess the primary endpoint of LOS. Stratified analyses were performed to check for the presence of effect modification or confounding. Two variables, presence of SIRs and CCI score (0-1 vs. $\geq 2$ ), were evaluated in stratified analyses. A multivariate analysis was performed using generalized linear regression with gamma and loglink to determine the adjusted LOS when controlling for baseline differences between the two groups. 


\section{Results}

During the study period, 122 patients met the study criteria, 61 patients received oritavancin, while the remaining 61 patients received the standard of care with vancomycin. Comparisons of baseline characteristics between the oritavancin group and the standard of care group are shown in Table 1. The two groups had similar demographic characteristics with a few exceptions. The standard of care group had a greater portion of patients with BMI $\geq 30 \mathrm{~kg} /$ $\mathrm{m}^{2}$ (30 vs $19 ; p<0.01$ ), but there was no statistical difference between the groups in the average BMI for all patients (31.9 vs $29.9 ; p=0.27$ ) or average body weight $(95.39 \mathrm{~kg}$ vs $88.2 \mathrm{~kg} ; p=0.22$ ). The standard of care group also had a higher number of diabetic patients (18 vs $6 ; p<0.01)$. In addition, it had a higher mean CCI ( 1.1 vs $0.54 ; p<0.01)$, likely due to the increased number of patients with peripheral vascular disease ( 16 vs $3 ; p<0.01$ ), since there was no difference between the groups in the number of patients with preexisting hepatitis, renal insufficiency, or an immunocompromised state. The standard of care group had more febrile patients ( 10 vs $3 ; p=0.02$ ), but there was no significant difference between the groups in the number of patients who met the SIRS criteria ( 16 vs $10 ; p=0.08$ ). The standard of care group also had a lower proportion of patients with IV drug abuse (IVDA) (12 vs. $21 ; p<0.01)$.
Table 1 Comparison of baseline demographics and clinical features between the pilot and standard of care groups

\begin{tabular}{|c|c|c|c|}
\hline & Pilot group $(n=61)$ & $\begin{array}{l}\text { Standard of care } \\
\text { group }(n=61)\end{array}$ & $P$ value \\
\hline \multicolumn{4}{|l|}{ Age (years) } \\
\hline Mean (SD) & $41.8(14.9)$ & $47.16(14.3)$ & 0.05 \\
\hline$\geq 65$ years $(n[\%])$ & $7(11.5)$ & $6(9.8)$ & 0.69 \\
\hline \multicolumn{4}{|l|}{ Gender $(n[\%])$} \\
\hline Male & $36(59)$ & $41(67.2)$ & 0.17 \\
\hline \multicolumn{4}{|l|}{ Race $(n[\%])$} \\
\hline White & $51(83.6)$ & $49(80.3)$ & 0.52 \\
\hline Black & $2(3.3)$ & $4(6.6)$ & 0.3 \\
\hline Other & $8(13.1)$ & $8(13.1)$ & 1 \\
\hline \multicolumn{4}{|l|}{ Body weight (kg) } \\
\hline Mean (SD) & $88.2(31.8)$ & $95.39(31.5)$ & 0.216 \\
\hline \multicolumn{4}{|l|}{ Body mass index $\left(\mathrm{kg} / \mathrm{m}^{2}\right)$} \\
\hline Mean (SD) & $29.9(10.5)$ & $31.9(9.3)$ & 0.267 \\
\hline$<25 \mathrm{~kg} / \mathrm{m}^{2}(n[\%])$ & $21(34.4)$ & $15(24.6)$ & 0.06 \\
\hline$\geq 30 \mathrm{~kg} / \mathrm{m}^{2}(n[\%])$ & $19(31.1)$ & $30(49.2)$ & $<0.01$ \\
\hline \multicolumn{4}{|l|}{ Past medical history $(n[\%])$} \\
\hline Diabetes mellitus & $6(9.8)$ & $18(29.5)$ & $<0.01$ \\
\hline Hepatitis & $6(9.8)$ & $7(11.5)$ & 0.17 \\
\hline Immunocompromised & $1(1.6)$ & $1(1.6)$ & 1 \\
\hline Renal insufficiency & $6(9.8)$ & $5(8.2)$ & 0.67 \\
\hline Severe peripheral vascular conditions & $3(4.9)$ & $16(26.2)$ & $<0.01$ \\
\hline IV drug abuse & $21(34.4)$ & $12(19.7)$ & $<0.01$ \\
\hline \multicolumn{4}{|l|}{ Charlson Comorbidity Index (CCI) } \\
\hline Mean (SD) & $0.54(0.93)$ & $1.1(1.27)$ & $<0.01$ \\
\hline \multicolumn{4}{|c|}{ Confirmed pathogen during admission $(n[\%])$} \\
\hline S. aureus & $5(33.3)$ & $18(41.9)$ & 0.08 \\
\hline MSSA & $1(6.7)$ & 5 (11.6) & 0.13 \\
\hline MRSA & $4(26.7)$ & $13(30.2)$ & 0.45 \\
\hline Other & $2(13.3)$ & $11(25.6)$ & $<0.01$ \\
\hline No cultures & $46(75.4)$ & $18(29.5)$ & $<0.01$ \\
\hline \multicolumn{4}{|l|}{ Patient presentation $(n[\%])$} \\
\hline Febrile & $3(4.9)$ & $10(16.4)$ & 0.02 \\
\hline Meeting SIRS criteria & $10(16.4)$ & $16(26.2)$ & 0.08 \\
\hline
\end{tabular}

IV intravenous, MRSA methicillin-resistant Staphylococcus aureus, MSSA methicillin-sensitive Staphylococcus aureus, $S D$ standard deviation, SIRS systemic inflammatory response syndrome 
Table 2 Comparison of outcomes between the pilot and standard of care groups
Fig. 1 Kaplan-Meier analysis of length of stay (measured in hours) for the pilot and standard of care groups. LOS length of stay (defined as the total time in hours from presentation to the emergency department until discharge home, including the time spent in the observation unit or inpatient unit)
Pilot group $(n=61)$

Standard of care group $p$ value $(n=61)$

\begin{tabular}{llll}
\hline $\begin{array}{l}\text { LOS, measured in hours } \\
\text { Mean (SD) }\end{array}$ & $19.5(20.7)$ & $85.98(50.34)$ & $<0.01$ \\
$\begin{array}{l}\text { 30-day inpatient readmission }(n[\%]) \\
\text { All cause }\end{array}$ & $6(9.8)$ & $6(9.8)$ & 1 \\
ID related & $5(8.2)$ & $3(5.9)$ & 0.24 \\
Recurrent ABSSSI & $2(3.3)$ & $3(5.9)$ & 0.55 \\
30-day ED return $(n[\%])$ & & & \\
All cause & $15(24.6)$ & $18(29.5)$ & 0.40 \\
ID related & $7(11.5)$ & $4(6.6)$ & 0.12 \\
Recurrent ABSSSI & $4(6.6)$ & $3(5.9)$ & 0.55 \\
Wound care & $2(3.3)$ & $4(6.6)$ & 0.30 \\
\hline
\end{tabular}

$A B S S I$ acute bacterial skin and skin structure infection, $E D$ emergency department, $I D$ infectious disease, $L O S$ length of stay, $S D$ standard deviation

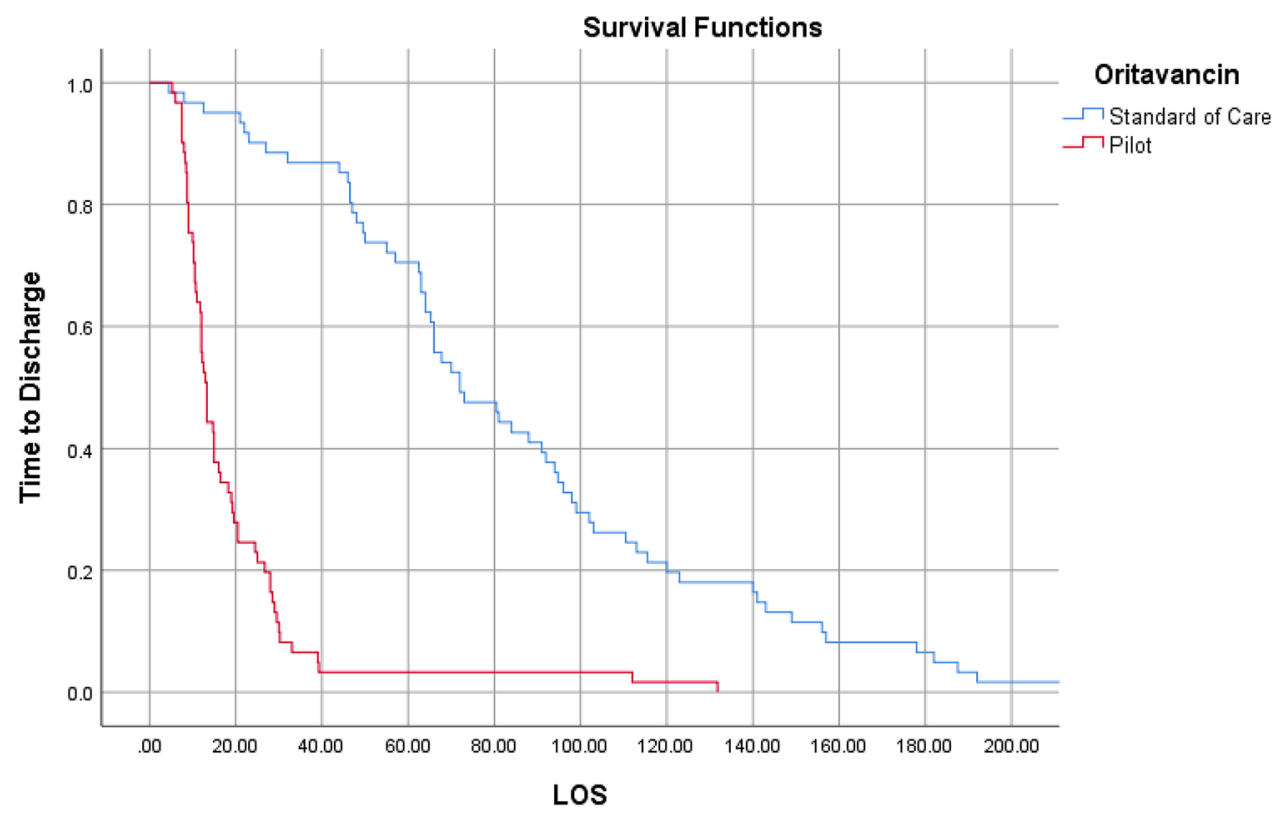

With regards to microbiologic data, the standard of care group had more patients with positive cultures. This was likely because more cultures were drawn in the standard of care group than in the pilot group (no cultures drawn: 18 vs $46 ; p<0.01)$. There was no statistical difference between the groups in the rate of $S$. aureus infection, nor when the infections were categorized into methicillin-sensitive Staphylococcus aureus (MSSA) or methicillin-resistant Staphylococcus aureus (MRSA) infections. There was a higher incidence of positive cultures due to other infections ( 11 vs $2 ; p<0.01$ ) in the standard of care group, which may be due to the small sample size of cultures in the pilot group.

Outcome comparisons are shown in Table 2. Administration of oritavancin in the ED and OBS (pilot group) was associated with a decreased mean LOS relative to the standard of care group (19.5 vs. $85.98 \mathrm{~h}, p<0.01)$. Figure 1 shows the results of the Kaplan-Meier analysis, which demonstrated a statistically significant decrease in median LOS for the pilot group (13.25 vs $72 \mathrm{~h}$, respectively, $p<0.01)$. Three patients in the pilot group were hospitalized after they were administered oritavancin in the OBS, which deviated from the pilot protocol and may have led to a longer average LOS. A multivariate regression analysis adjusting for baseline variables showed that the average LOS for the pilot group was still decreased compared to the standard of care group (37.73 vs $159.41, p<0.01)$. Additionally, a stratified analysis resulted in a statistically significant decrease in LOS for patients with and without positive SIRS criteria, and in patients with $\mathrm{CC} \leq 1$ and $\mathrm{CCI}>1$ (Table 3 ). 
Table 3 Comparison of outcomes between the pilot and standard of care groups stratified by the presence of systemic inflammatory response syndrome (SIRS) and the Charlson Comorbitidy Index (CCI) score

\begin{tabular}{|c|c|c|c|}
\hline \multicolumn{4}{|l|}{ Presence of SIRs } \\
\hline & Pilot & Standard of care & $p$-value \\
\hline & $(n=10)$ & $(n=16)$ & \\
\hline Mean (SD) LOS, measured in $\mathrm{h}$ & $13.6(8)$ & $78.2(38.5)$ & $<0.01$ \\
\hline 30-Day readmission ( $n[\%])$ & $1(10)$ & $1(6.3)$ & \\
\hline 30-Day ED return $(n[\%])$ & $1(10)$ & $2(14.3)$ & \\
\hline \multicolumn{4}{|l|}{ Absence of SIRs } \\
\hline & Pilot & Standard of care & $p$-value \\
\hline & $(n=51)$ & $(n=45)$ & \\
\hline Mean (SD) LOS, measured in $\mathrm{h}$ & $20.6(22.2)$ & $88.8(53.7)$ & $<0.01$ \\
\hline 30-Day readmission $(n[\%])$ & $5(9.8)$ & $5(11.1)$ & \\
\hline 30-Day ED return $(n[\%])$ & $14(27.5)$ & $16(35.6)$ & \\
\hline \multicolumn{4}{|l|}{ CCI score $\leq 1$} \\
\hline & Pilot & Standard of care & $p$-value \\
\hline & $(n=55)$ & $(n=43)$ & \\
\hline Mean (SD) LOS, measured in $\mathrm{h}$ & $20.3(21.6)$ & $85.4(48.6)$ & $P<0.01$ \\
\hline 30-Day readmission $(n[\%])$ & $5(9.1)$ & $4(9.3)$ & \\
\hline 30-Day ED return $(n[\%])$ & $13(23.6)$ & $15(34.9)$ & \\
\hline \multicolumn{4}{|l|}{ CCI score $>1$} \\
\hline & Pilot & Standard of care & $p$-value \\
\hline & $(n=6)$ & $(n=18)$ & \\
\hline Mean (SD) LOS, measured in $\mathrm{h}$ & $11.4(2.2)$ & $86.2(51.1)$ & $<0.01$ \\
\hline 30-Day Readmission ( $n[\%]$ ) & $1(16.7)$ & $2(11.1)$ & \\
\hline 30-Day ED Return ( $n[\%])$ & $2(33.3)$ & $3(16.7)$ & \\
\hline
\end{tabular}

$E D$ emergency department, $h$ hours, $L O S$ length of stay, $n$ number, $S D$ standard deviation

The rates of subsequent all-cause 30-day readmissions were identical for the pilot and standard of care groups (6 patients in each group, 9.8\% readmission rate), and the recurrent ABSSSI 30-day readmission rates were also comparable. In the oritavancin pilot, two patients returned due to a worsening of the infection-one after refusing wound wrapping after incision and drainage, and the other after reporting improvements for the first 2 weeks before symptoms returned. Two additional patients were asked to return due to positive blood cultures for $S$. aureus, since the pilot was only approved for the treatment of ABSSSI. Similarly, return ED visits within 30 days were comparable between groups; however, some patients in the oritavancin pilot were asked to return to the ED for wound care. These patients were included in our 30-day ED return data as they are included in our hospital's metrics.

Nineteen patients from the pilot group were reached via phone for follow-up from a total of 36 patients that had documented follow-up upon chart review. Three patients
Table 4 Financial outcomes of the oritavancin pilot

Cost savings due to hospital admission avoidance $(N=55)^{*}$

Days 227

Cost saved (US dollars)

$\$ 308,000$

*6 of 61 patients were readmitted within 30 days to the inpatient unit after receipt of oritavancin and discharge from the emergency department. Savings were calculated using an average cost of $\$ 5600$ per cellulitis diagnosis-related group and an average length of stay of 4.13 days (internal data)

returned to the ED before a call was placed, and the remaining patients did not have any documentation of a follow-up phone call. All 19 patients reported an improvement, and 17 of those had no further complaints. Two patients still had some symptoms, though they reported that those symptoms were improved by their visits. Those patients were instructed to follow up with their primary care provider or to return to 
the ED if the symptoms persisted or worsened. Neither of those patients returned to the ED.

Cost savings were found to be $\$ 308,000$ USD based on the avoidance of 227 days of inpatient admissions at initial presentation (Table 4). These savings were derived from the average cost of care for cellulitis patients and the corresponding LOS, and do not include liability or iatrogenicity.

\section{Discussion}

Patients with ABSSSIs are largely treated with oral antibiotics in the outpatient setting or with IV antibiotics as inpatients, who are often dosed multiple times a day for an extended duration. For patients who fail outpatient treatment, the next step is typically hospital inpatient admission to receive IV treatment for multiple days [15]. This practice places a significant cost burden on the patient and the utilization of healthcare resources, as each ABSSSI-related hospital admission is estimated to cost between $\$ 6000$ and $\$ 20,000$ USD $[5,16,17]$. Due to the significant hospitalization costs associated with ABSSSI, and internally collected data suggesting that many ABSSSI patients are admitted unnecessarily, a pilot program was implemented at AHEO to administer oritavancin in the ED or OBS as a measure to avert hospital inpatient admissions in clinically stable ABSSSI patients.

Overall, the results suggest that using oritavancin in the ED or OBS unit to facilitate discharge to home may result in a decreased LOS relative to standard of care practices, although causality could not be established in this study due to its design. The findings also suggest that the use of oritavancin may not result in an increased rate of ED return visits. Although definitive conclusions cannot be drawn from the study, the collective results suggest that the primary financial benefit was was cost savings from reduced inpatient admission among the pilot group.

The findings from this study are largely comparable to those in several recent publications of real-world evidence. In an assessment of 67 patients who received oritavancin at a community hospital, no readmissions and $8 \mathrm{ED}$ return visits were observed within 14 days of discharge [18]. Similarly, the 30-day subsequent readmission rate was reported to be $6.1 \%$ in an evaluation of oritavancin for ABSSSI patients across three hospital-based outpatient infusion centers [19]. In our study, the observed 30-day readmission rate was slightly higher $(9.8 \%)$, but this may have been due to the aggressive mandated pharmacy callback system, which encouraged patients to return to the hospital if symptoms were not completely resolved. However, this readmission rate may also be representative of the patient population in this area of the city, as it was comparable to the readmission rate for the standard of care group.
Several aspects should be noted when interpreting these findings. First, this was a single-center retrospective cohort study, and it is therefore subject to all the limitations and biases associated with this design. Most notably, we cannot fully gauge the impact of prescribing bias on the observed findings. Inclusion in the pilot was dependent on the physician's knowledge of, and subsequent comfort with, the oritavancin pilot. Based on the author's interactions with the prescribers, many providers did not commonly prescribe oritavancin due to a lack of knowledge of the pilot or because they had forgotten about it. A total of 38 providers utilized the pilot protocol for the 61 patients included in the study. None of the prescriber(s) accounted for the majority of the orders. Twenty-three of these providers were ED providers, while the remainder were OBS or ID specialists. Inclusion in the standard of care cohort required the application of the same inclusion/exclusion criteria as used during the pilot, as this ensured the recruitment of patients who could have been included in the pilot had a more aggressive strategy been used to include every qualifying patient. Furthermore, oritavancin was primarily used in patients who were perceived as potentially noncompliant (IVDAs, the homeless, and those with social issues). It is unclear if or how providers' treatment preferences (i.e., prescribing bias) influenced the result.

The standard of care cohort group had higher CCI scores and more complicating comorbidities such as diabetes and peripheral vascular disease, and this may have contributed to their hospital LOS. However, no differences between the groups were observed in the stratified or multivariate analyses that looked at patients with varying CCI scores, providing further credence that oritavancin may decrease LOS. Furthermore, the observed LOS in the standard of care group was approximately 4 days, which is consistent with literature estimates $[5,16,17]$ and the internal quality assurance data collected prior to initiating the oritavancin pilot. Finally, unknown readmissions may have occurred outside the system. Subsequent readmission data were collected from within the same hospital system, and it is possible that some patients may have sought treatment at their primary care office or from another hospital system. Despite this, any missed subsequent readmissions were just as likely to occur in the pilot and standard of care treatment groups, so they would not have significantly altered the findings.

In conclusion, this study suggests that oritavancin may be administered in the ED or OBS to prevent unnecessary hospital inpatient admissions of stable ABSSSI patients without incurring increased readmission rates. Early directed oritavancin use was not found to result in increased 30-day subsequent readmissions and 30-day return ED visits relative to standard inpatient treatment with vancomycin. Additionally, the pilot program was associated with reduced costs. Patients in other studies have reported a preference for receiving 
ABSSSI treatment in the outpatient setting rather than hospitalization [20]. Oritavancin has the potential to shift ABSSSI care from the inpatient to the outpatient setting, which could improve the patient's experience and satisfaction. However, as with all retrospective single-site studies, our findings should be interpreted cautiously and verified with a multicenter prospective study. Patient experience data should be included in all future studies as part of the benefit-risk assessment, given the growing focus on patient-centeredness in health care.

\section{Acknowledgements None.}

Author contributions All authors had a role in study design, conceiving, and writing the manuscript. According to the guidelines of the International Committee of Medical Journal Editors (ICMJE, www. icmje.org), all authors met the criteria for authorship and no deserving authors have been omitted.

\section{Compliance with Ethical Standards}

Funding No funding was provided for this study or manuscript preparation. This manuscript is part of a supplement wholly funded by Melinta Therapeutics.

Conflict of Interest TL receives consulting and speaker fees from Melinta Therapeutics. SA, SB, BH, AM, and KR have no conflicts to declare.

Open Access This article is licensed under a Creative Commons Attribution-NonCommercial 4.0 International License, which permits any non-commercial use, sharing, adaptation, distribution and reproduction in any medium or format, as long as you give appropriate credit to the original author(s) and the source, provide a link to the Creative Commons licence, and indicate if changes were made. The images or other third party material in this article are included in the article's Creative Commons licence, unless indicated otherwise in a credit line to the material. If material is not included in the article's Creative Commons licence and your intended use is not permitted by statutory regulation or exceeds the permitted use, you will need to obtain permission directly from the copyright holder. To view a copy of this licence, visit http://creativecommons.org/licenses/by-nc/4.0/.

\section{Appendix A}

AdventHealth oritavancin criteria for use policy. Inclusion criteria:

- Treatment of known or suspected ABSSSI caused by susceptible isolates of gram-positive pathogens with at least one of the following:

- Failed oral therapy or not a candidate for oral therapy

- Suspect patient noncompliance or poor patient reliability.

Exclusion criteria:

- Hemodynamically unstable, altered mental status, acute organ injury

- Suspicion of systemic infection including bacteremia, osteomyelitis, or endocarditis

- Chronic infection

- Need for surgical intervention, necrotizing fasciitis, or orbital cellulitis

- Suspicion of gram-negative organism or failed outpatient IV gram-positive therapy

- Other factors requiring inpatient admission

- Diabetic ketoacidosis or hyperosmolar hyperglycemic state (HHS)

- Concurrent use of intravenous unfractionated heparin sodium within $120 \mathrm{~h}$ (5 days).

\section{Appendix B}

See Table 5.

Table 5 ICD-10 diagnostic codes consistent with Orbactiv ${ }^{\circledR}$ (oritavancin) indication

\begin{tabular}{ll}
\hline ICD-10-CM diagnosis codes & Diagnosis \\
\hline Staphylococcus & \\
A49.01 & Methicillin-susceptible Staphylococcus aureus, unspecified site \\
A49.02 & Methicillin-resistant Staphylococcus aureus infection, unspecified site \\
B95.62 & Staphylococcus aureus as the cause of diseases classified elsewhere (methicillin resistant) \\
B95.61 & Staphylococcus aureus as the cause of diseases classified elsewhere (methicillin susceptible) \\
B95.8 & Unspecified Staphylococcus as the cause of diseases classified elsewhere \\
Streptococcus & \\
A49.1 & Streptococcus infection, unspecified site \\
B95.0-B95.2, B95.4 & Streptococcus, as the cause of disease classified elsewhere \\
\hline
\end{tabular}


Table 5 (continued)

\begin{tabular}{|c|c|}
\hline ICD-10-CM diagnosis codes & Diagnosis \\
\hline \multicolumn{2}{|l|}{ Other infections } \\
\hline A46 & Erysipelas \\
\hline L08.0-L08.1, L08.81-L08.89, L08.9 & Other local infections of skin and subcutaneous tissue \\
\hline \multicolumn{2}{|l|}{ Cellulitis } \\
\hline L03.211 & Cellulitis of face \\
\hline $\mathrm{K} 12.2$ & Cellulitis and abscess of mouth \\
\hline H05.011-H05.019 & Cellulitis of orbit, abscess of orbit \\
\hline H60.10-H60.13 & Cellulitis of external ear \\
\hline $\mathrm{J} 34.0$ & Cellulitis and abscess of external nose \\
\hline L03.221 & Cellulitis of neck \\
\hline L03.113-L03.114 & Cellulitis of upper limb \\
\hline L03.111-L03.114 & Cellulitis of axilla and upper limb \\
\hline L03.011-L03.019 & Cellulitis of finger \\
\hline N61 & Inflammatory disorders of breast (includes cellulitis/abscess breast) \\
\hline L03.311-L03.316, L03.319 & Cellulitis of trunk \\
\hline L03.317 & Cellulitis of buttock \\
\hline L03.119 & Cellulitis of unspecified part of limb \\
\hline L03.115-L03.116 & Cellulitis of lower limb \\
\hline $\mathrm{N} 48.22$ & Cellulitis of corpus cavernosum and penis \\
\hline L03.031-L03.039 & Cellulitis of toe \\
\hline L03.811-L03.818 & Cellulitis of other sites \\
\hline L03.90 & Cellulitis, unspecified \\
\hline \multicolumn{2}{|l|}{ Abscess } \\
\hline L02.01 & Cutaneous abscess of face \\
\hline H00.031-H00.039 & Abscess and furuncle of eyelid \\
\hline H60.00-H60.03 & Abscess of external ear \\
\hline $\mathrm{K} 12.2$ & Submandibular abscess \\
\hline L02.11 & Cutaneous abscess of neck \\
\hline L02.411-L02.414 & Cutaneous abscess of axilla and upper limb \\
\hline L02.511-L02.519 & Cutaneous abscess of hand \\
\hline L02.211-L02.219 & Cutaneous abscess of trunk \\
\hline L02.31 & Cutaneous abscess of buttock \\
\hline L02.419 & Cutaneous abscess of limb, unspecified \\
\hline L02.415-L02.416 & Cutaneous abscess of lower limb \\
\hline L02.611-L02.619 & Cutaneous abscess of foot \\
\hline $\mathrm{N} 48.21$ & Abscess of corpus cavernosum and penis \\
\hline N76.4 & Abscess of vulva \\
\hline K61.0-K61.4 & Abscess of anal and rectal regions \\
\hline L02.811-L02.818 & Cutaneous abscess of other sites \\
\hline L02.91 & Cutaneous abscess, unspecified \\
\hline \multicolumn{2}{|l|}{ Furuncle } \\
\hline L02.02 & Furuncle of face \\
\hline L02.12 & Furuncle of neck \\
\hline L02.421-L02.424 & Furuncle of axilla, upper limb \\
\hline L02.521-L02.529 & Furuncle of hand \\
\hline L02.221-L02.229 & Furuncle of trunk \\
\hline L02.32 & Furuncle of buttock \\
\hline L02.429 & Furuncle of limb, unspecified \\
\hline L02.425-L02.426 & Furuncle of lower limb \\
\hline L02.621-L02.629 & Furuncle of foot \\
\hline
\end{tabular}


Table 5 (continued)

\begin{tabular}{ll}
\hline ICD-10-CM diagnosis codes & Diagnosis \\
\hline L02.821-L02.828 & Furuncle of other sites \\
L02.92 & Furuncle, unspecified \\
Carbuncle & \\
L02.03 & Carbuncle of face \\
J34.0 & Carbuncle and furuncle of external nose \\
L02.13 & Carbuncle of neck \\
L02.431-L02.434 & Carbuncle of axilla, upper limb \\
L02.531-L02.539 & Carbuncle of hand \\
L02.231-L02.239 & Carbuncle of trunk \\
L02.33 & Carbuncle of buttock \\
L02.439 & Carbuncle of limb, unspecified \\
L02.435-L02.436 & Carbuncle of lower limb \\
L02.631-L02.639 & Carbuncle of foot \\
L02.831-L02.838 & Carbuncle of other sites \\
L02.93 & Carbuncle, unspecified \\
\hline
\end{tabular}

\section{References}

1. Melinta Therapeutics. Prescribing information for Orbactiv (oritavancin). https://www.orbactiv.com/pdfs/orbactiv-prescribing-infor mation.pdf.

2. Van Bambeke F. Lipoglycopeptide antibacterial agents in gram-positive infections: a comparative review. Drugs. 2015;75(18):2073-95.

3. Corey GR, Kabler H, Mehra P, Gupta S, Overcash JS, Porwal A, et al. Single-dose oritavancin in the treatment of acute bacterial skin infections. N Engl J Med. 2014;370(23):2180-90.

4. Corey GR, Good S, Jiang H, Moeck G, Wikler M, Green S, et al. Single-dose oritavancin versus 7-10 days of vancomycin in the treatment of gram-positive acute bacterial skin and skin structure infections: the SOLO II noninferiority study. Clin Infect Dis. 2015;60(2):254-62.

5. Lodise TP, Fan W, Sulham KA. Hospital admission patterns in adult patients with skin and soft tissue infections: Identification of potentially avoidable hospital admissions through a retrospective database analysis. Hosp Pract. 2015;43(3):137-43.

6. Dryden M, Saeed K, Townsend R, Winnard C, Bourne S, Parker $\mathrm{N}$, et al. Antibiotic stewardship and early discharge from hospital: impact of a structured approach to antimicrobial management. J Antimicrob Chemother. 2012;67(9):2289-96.

7. Lodise TP, Fan W, Sulham KA. Hospital admission patterns in adult patients with skin and soft tissue infections: identification of potentially avoidable hospital admissions through a retrospective database analysis. Hosp Pract. 2015;43(3):137-43.

8. Lodise TP, Fan W, Sulham KA. Economic impact of oritavancin for the treatment of acute bacterial skin and skin structure infections in the emergency department or observation setting: cost savings associated with avoidable hospitalizations. Clin Ther. 2016;38(1):136-48.

9. Lodise TP, Redell M, Armstrong SO, Sulham KA, Corey GR. Efficacy and safety of oritavancin relative to vancomycin for patients with acute bacterial skin and skin structure infections (ABSSSI) in the outpatient setting: results from the SOLO clinical trials. Open Forum Infect Dis. 2017;4(1):274.

10. Redell M, Moeck G, Lucasti C, Durso S, Kennedy C, Fusaro K, et al. A real-world patient registry for oritavancin demonstrates efficacy and safety consistent with the phase 3 SOLO program. Open Forum Infect Dis. 2018;5(6):051.
11. Center for Drug Evaluation and Research. Guidance for industry acute bacterial skin and skin structure infections: developing drugs for treatment. https://www.fda.gov/files/Acute-BacterialSkin-and-Skin-Structure-Infections---Developing-Drugs-for-Treat ment.pdf.

12. Bone RC, Balk RA, Cerra FB, Dellinger RP, Fein AM, Knaus WA, et al. Definitions for sepsis and organ failure and guidelines for the use of innovative therapies in sepsis. The ACCP/ SCCM Consensus Conference Committee. American College of Chest Physicians/Society of Critical Care Medicine. Chest. 1992;101(6):1644-55.

13. Charlson ME, Pompei P, Ales KL, MacKenzie CR. A new method of classifying prognostic comorbidity in longitudinal studies: development and validation. J Chronic Dis. 1987;40(5):373-83.

14. Cockcroft DW, Gault MH. Prediction of creatinine clearance from serum creatinine. Nephron. 1976;16(1):31-41.

15. Stevens DL, Bisno AL, Chambers HF, Dellinger EP, Goldstein EJ, Gorbach SL, et al. Practice guidelines for the diagnosis and management of skin and soft tissue infections: 2014 update by the Infectious Diseases Society of America. Clin Infect Dis. 2014;59(2):147-59.

16. Agency for Healthcare Research and Quality. Healthcare Costs and Utilization Project (HCUPnet). https://hcupnet.ahrq.gov/query/ey. Accessed March 2019.

17. Suaya JA, Mera RM, Cassidy A, O'Hara P, Amrine-Madsen H, Burstin S, et al. Incidence and cost of hospitalizations associated with Staphylococcus aureus skin and soft tissue infections in the United States from 2001 through 2009. BMC Infect Dis. 2014;14:296.

18. Co D, Roebuck L, VanLandingham J. Evaluation of oritavancin use at a community hospital. Hosp Pharm. 2018;53(4):272-6.

19. Estrada S, Lodise TT, GS, Delaportas D. The real world economic and clinical management of adult patients with skin and soft tissue (SSTI) infections with oritavancin: Data from two multi-center observational cohort studies. 2019.

20. Almarzoky Abuhussain SS, Burak MA, Kohman KN, Jacknin G, Tart SB, Hobbs ALV, et al. Patient preferences for treatment of acute bacterial skin and skin structure infections in the emergency department. BMC Health Serv Res. 2018;18(1):932.

21. Melinta Therapeutics. ICD-10 diagnostic codes consistent with ORBACTIV $^{\circledR}$ (oritavancin) indication. https://www.orbactiv.com/ pdfs/orbactiv-icd10-codes.pdf. 